\section{Effect of Short-Circuiting in Electrical Discharge Machining of Carbon Fiber Reinforced Plastics*}

\author{
Akihiro ITO**, Shinya HAYAKAWA***, Fumihiro ITOIGAWA**** \\ and Takashi NAKAMURA**** \\ **Department of mechanical engineering, Nagoya Institute of Technology, \\ Gokiso-cho, Showa-ku, Nagoya 466-8555, Japan \\ E-mail: ciq16511@stn.nitech.ac.jp \\ ***Department of mechanical engineering, Nagoya Institute of Technology, \\ Gokiso-cho, Showa-ku, Nagoya 466-8555, Japan \\ ****Department of mechanical engineering, Nagoya Institute of Technology, \\ Gokiso-cho, Showa-ku, Nagoya 466-8555, Japan
}

\begin{abstract}
In this paper, we investigate the effects of short-circuiting on machining in order to discuss material removal in EDM of CFRP. Sinking EDM of CFRP using deionized water was carried out, and waveforms of gap voltage and current, pulse frequency and servo feed fluctuation, were measured for classifying machining situations, electrical discharge and short-circuiting. The cause of short-circuiting and the relationship between the short-circuiting ratio and material removal rate were investigated. In addition, the potential drop and temperature of short-circuiting carbon fibers were estimated with a simple model. Experimental results show that short-circuiting due to frayed carbon fibers frequently occurs in sinking EDM of CFRP. It is found that the short-circuiting contributes to the material removal in EDM of CFRP.
\end{abstract}

Key words: Electrical Discharge Machining (EDM), Carbon Fiber Reinforced Plastics (CFRP), Deionized Water, Short-Circuiting, Material Removal

\section{Introduction}

Carbon fiber reinforced plastics (CFRP) has exceptional characteristics, such as high strength, high rigidity, high resistance to impact, high corrosion resistance, and light weight. Because of these positive characteristics, CFRP is one of the promising materials in the aircraft industry and automobile industry.

However, CFRP is known to be a material that is hard to process. In conventional machining methods of CFRP, such as drilling, milling and turning, cutting tools wear egregiously and chipping of the tools often occurs during machining owing to the high strength of carbon fibers. Furthermore, machined CFRP also has problems, such as delamination, splintering and the presence of burrs owing to its composite structure ${ }^{(1-2)}$.

Electrical discharge machining (EDM) can be used for the machining of materials that are difficult to process. For example, it is reported that EDM is applicable to the machining of several insulating ceramics such as $\mathrm{Si} 3 \mathrm{~N} 4, \mathrm{SiC}$, and $\mathrm{ZrO} 2{ }^{(3)}$. If $\mathrm{EDM}$ can be applied to the machining of CFRP, the problems that disturb CFRP machining in conventional methods can be avoided. EDM of CFRP has been carried out by the authors ${ }^{(4)}$ and Wang et al. ${ }^{(5)}$. Wang et al. investigated the EDM properties, such as material removal rate, electrode
*Received 11 Jan., 2012 (No. 12-0015) [DOI: 10.1299/jamdsm.6.808]

Copyright $\odot 2012$ by JSME 
wear ratio and surface roughness, of CFRP in kerosene. They reported that the EDM performance for CFRP becomes unstable under a high discharge current condition and that no-electrode-wear EDM for CFRP is possible under appropriate conditions. The authors also reported on EDM properties of CFRP. In our experiments, deionized water was used as the machining liquid in order to avoid the deterioration of plastics owing to oil. It was found for sinking EDM that short-circuiting was observed frequently. Although short-circuiting does not contribute to material removal in EDM of metal, there is some possibility that short-circuiting contributes to material removal in EDM of CFRP because CFRP is composed of carbon fibers and resin. Saeki et al. ${ }^{(6)}$ reported for the case of high electrical resistance material that Joule heating generated in the material affects the material removal.

In this paper, we investigate the effects of short-circuiting on marching and their causes in order to discuss material removal in EDM of CFRP.

\section{Effect of Short-Circuiting}

\subsection{Classification of Machining Situations}

The typical situations observed during in sinking EDM of CFRP can be classified into two cases, situation $\mathrm{A}$ and situation $\mathrm{B}$, as shown in figure 1. In the case of machining situation A, intense emission of light and high-frequency sound are generated intermittently from the machining domain. On the other hand, in the case of machining situation B, light is emitted from the gap space and machining sound is generated, that are similar to those in conventional EDM of metal.

In order to discuss machining phenomena occurring in the above cases and their influence on EDM of CFRP, gap voltage and discharge current were investigated. Table 1 shows the material properties of the CFRP used in this experiment. The CFRP is a flat plate $11.5 \mathrm{~mm}$ thick made by laminating prepregs of carbon fiber cloth with various fiber orientations. The fiber content ratio of the CFRP is approximately $90 \%$, which is estimated in a prepreg of a section perpendicular to the fiber orientation. Sinking EDM using deionized water was carried out in the direction parallel to the prepreg, as shown in figure 2. Table 2 lists the experimental conditions.

Figures 3(a) and 3(b) show the observed waveforms of gap voltage and discharge current for situations A and B, respectively. In the case of situation A, the fall of gap voltage and the rise of discharge current are steeper than those in situation B. In addition, the measured value of the peak discharge current and the gap voltage are approximately $20 \mathrm{~A}$ and $30 \mathrm{~V}$ in situation A, but, $12 \pm 1 \mathrm{~A}$ and $45-50 \mathrm{~V}$ in situation $\mathrm{B}$, respectively.

When several carbon fibers, prepared by fiberizing a carbon fiber cloth, are connected to the power supply of the sinking EDM and electrical current flows through them, an intense emission of light similar to the case of situation A was observed. This result indicates that the intense emission of light shown in figure 1(a) can be caused by Joule heating of carbon fibers. In the EDM process, an intense emission of light is often observed when electrical insulation in the gap is insufficiently recovered during pulse interval times; this is called arcing. However, the situation A is thought to be not arcing but short-circuiting

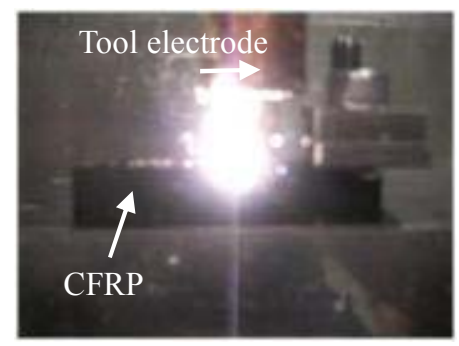

(a) Situation $\mathrm{A}$

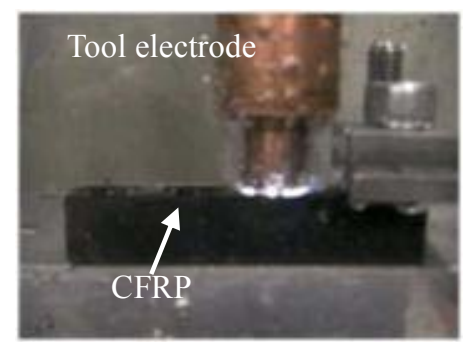

(b) Situation B

Fig. 1 Overall view of machining situations 
Table 1 Material properties in evaluating

the ratio of the short-circuiting

\begin{tabular}{c|c}
\hline Thickness & $11.50 \mathrm{~mm}$ \\
\hline Fiber orientation & $0^{\circ}, \pm 45^{\circ}, 90^{\circ}$ \\
\hline Fiber diameter & $8 \mu \mathrm{m}$ \\
\hline Table 2 Experimental conditions for evaluating \\
the ratio of the short-circuiting \\
\hline Tool electrode & $\mathrm{Cu}(\varphi 10 \mathrm{~mm})$ \\
\hline Polarity & Tool electrode $(+)$ \\
\hline Open circuit voltage & $85 \mathrm{~V}$ \\
\hline Discharge current & $24 \mathrm{~A}$ \\
\hline Discharge duration & $32 \mu \mathrm{s}$ \\
\hline Pulse interval time & $128 \mu \mathrm{s}$ \\
\hline Dielectric fluid & Deionized water \\
\hline
\end{tabular}

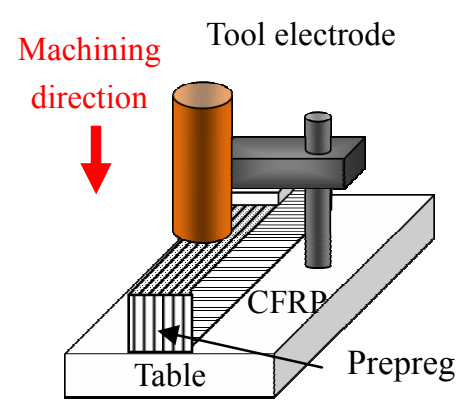

Fig. 2 Experimental setup for evaluating the ratio of short-circuiting

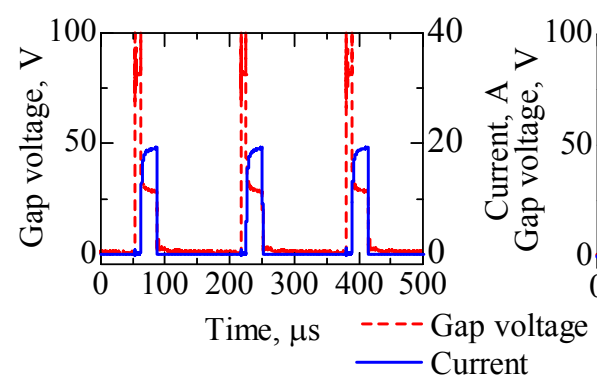

(a) Situation A

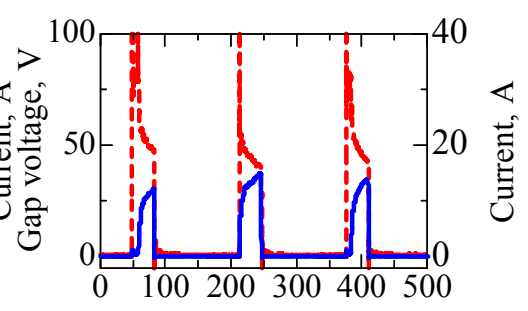

Time, $\mu$ s ----Gap voltage

(b) Situation B

Fig. 3 Observed waveforms of gap voltage and discharge current

by the carbon fibers because discharge delay time exists for each pulse. The fact that higher current and lower gap voltage are observed in the pulse duration compared with situation B, as shown in figure 3, supports the above conclusion.

On the other hand, the situation B is thought to be electrical discharge, because the overview is similar to that in machining of a metal. In situation A, the gap voltage of $30 \mathrm{~V}$ is thought to be the potential drop due to the internal resistance of the CFRP. The gap voltage in the pulse duration in situation B is higher approximately $20 \mathrm{~V}$ than that in situation A, which indicates a potential drop due to electrical discharge.

\subsection{Cause of the Short-Circuiting}

When CFRP was machined by sinking EDM for 20 minutes under the conditions shown in table 2, the average ratio of short-circuiting, namely, situation A, was $40 \%$, whereas that for metal was rare under the same conditions. Therefore, the factors of short-circuiting in the EDM of CFRP are discussed in this section.

One of the significant factors is carbon fibers that are exposed and fraying on the machined surface. Figure 4(a) shows the SEM image of the machined surface of CFRP under the machining conditions shown in table 2. It is found that the carbon fibers on the machined surface of CFRP are exposed and frayed because the melting temperature of the resin is much lower than the sublimation temperature of the carbon fiber, and the resin is removed selectively during EDM of CFRP. One of the probable causes of the short-circuiting is the frayed carbon fiber coming into contact with the tool electrode owing to the attractive force of the electric field during the discharge delay time.

Large carbon fiber debris is also thought to be a factor in short-circuiting. Figure 4(b) shows the debris floating in the machining liquid. The large pieces of carbon fibers, more than $500 \mu \mathrm{m}$ in length, are observed. Kunieda and Yanatori ${ }^{(7)}$ investigated debris particle 
Fraying carbon fibers

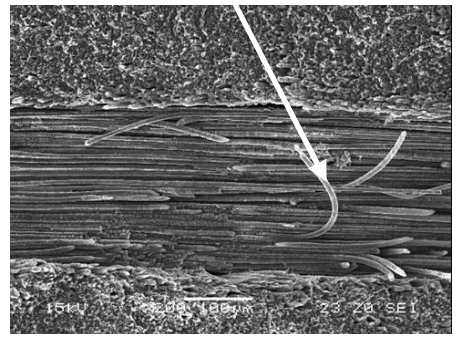

(a) Machined surface
Carbon fibers

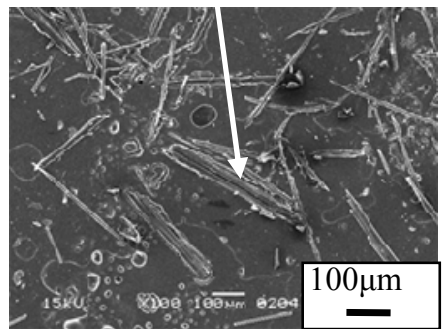

(b) Debris of carbon fibers

Fig. 4 Machined surface and debris in EDM of CFRP

motion caused by electric force and reported that debris particles form bridges between the anode and the cathode during the discharge delay time. If carbon fiber debris exists in the narrow gap and an electric field is applied to the gap, short-circuiting may occur because carbon fibers could easily form some bridges between the anode and the cathode.

\subsection{Contribution of Short-Circuiting to Machining}

In order to investigate the influence of short-circuiting on the material removal rate, the ratio of short-circuiting pulses to total pulses and servo feed fluctuation were investigated

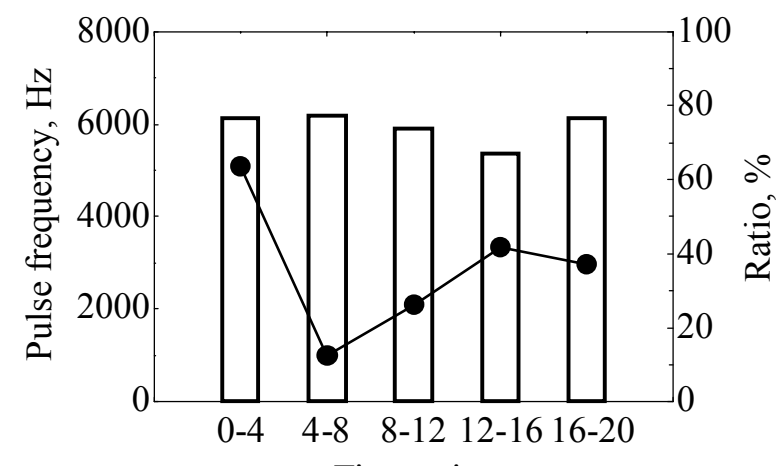

Time, $\min$

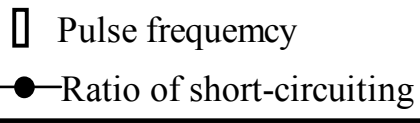

Fig. 5 Discharge frequency and ratio of short-circuiting

Average servo feed ratio

$0.13 \mathrm{~mm} / \mathrm{min} \quad 0.13 \mathrm{~mm} / \mathrm{min}$

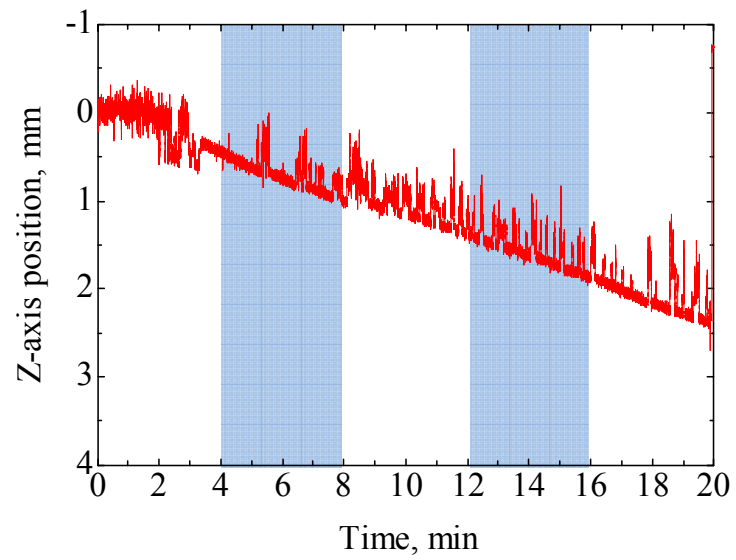

Fig. 6 Servo feed fluctuation 
under the machining conditions shown in table 2. The ratio of short-circuiting was defined as the ratio of the waveforms shown in figure 3(a). The servo feed fluctuation was measured using an electrical micrometer.

Figure 5 shows the obtained results of the average pulse frequency and the ratio of short-circuiting. The ratio of short-circuiting changes from $10 \%$ to $60 \%$ depending on machining time, whereas the average pulse frequency remains at approximately $6,000 \mathrm{~Hz}$. Figure 6 shows the measured result of servo feed fluctuations. It is found that the $z$-axis position of the tool electrode returns to the same line of the servo feed, after it is pulled up due to short-circuiting, except when it is pull up for a long time. To make a comparison between the average servo feed rate for machining time between 4 to 8 minutes when the average ratio of the short-circuiting is $10 \%$, and the value between 12 to 16 minutes when the average ratio is $40 \%$, it is found that the average servo feed rate is almost constant at $0.13 \mathrm{~mm} / \mathrm{min}$ in both cases in spite of the ratio of short-circuiting being varied from $10 \%$ to $40 \%$. This result indicates that short-circuiting contributes to material removal in EDM of CFRP, whereas it does not contribute to material removal in EDM of metal.

Saeki et al. (6) investigated the influence of the Joule heating of high-electrical-resistance material on the removal mechanics of EDM and report that Joule heating causes a temperature rise near the discharge spot, which affects the material removal. In sinking EDM of CFRP, resin is thought to be removed upon the rise in temperature caused by Joule heating of carbon fibers in the short-circuiting pulse.

\section{Estimation of Number of Short-Circuiting Carbon Fibers}

In order to verify the validity of the discussion mentioned in section 2 and to estimate how many carbon fibers come into contact with the tool electrode in short-circuiting, the potential drop and temperature of the short-circuiting carbon fibers are calculated. Figure 7 shows the calculation model used for this estimation. For simplicity, the carbon fibers $300 \mu \mathrm{m}$ in length undergo short-circuiting.

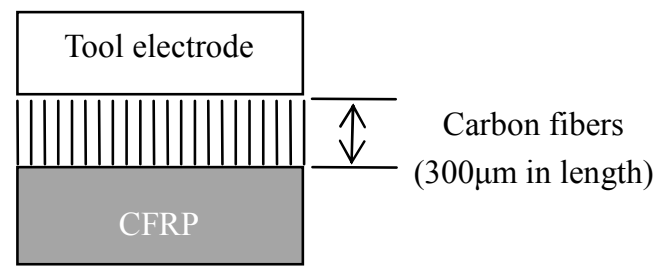

Fig. 7 Calculation model of short-circuiting due to carbon fibers

\subsection{Estimation using Potential Drop in Carbon Fibers}

In short-circuiting by carbon fibers, the relationship between the number of carbon fibers and the potential drop in the fibers is investigated in order to estimate how many carbon fibers undergo short-circuiting. Since the electric resistivity of a carbon fiber is $2 \times 10^{-5} \Omega \cdot \mathrm{m}^{(8)}$, the resistance of a carbon fiber $8 \mu \mathrm{m}$ in diameter and $300 \mu \mathrm{m}$ in length is estimated to be $120 \Omega$. When the short-circuit current is $20 \mathrm{~A}$, as shown in figure $3(\mathrm{a})$, the relationship between the number of carbon fibers in contacted with the tool electrode and the potential drop in the fibers can be obtained as shown in figure 8 .

If a gap voltage of $30 \mathrm{~V}$, as shown in figure $3(\mathrm{a})$, is assumed to be generated as a result of the potential drop in the carbon fibers, the necessary number of carbon fibers can be estimated to be 80 . Actually, the measured gap voltage, $30 \mathrm{~V}$, includes the potential drop generated in the body of the CFRP, and contact resistances between carbon fibers and the tool electrode and between CFRP and the machine table, in addition to the potential drop in short-circuiting carbon fibers. Therefore, the actual value of the potential drop in short-circuiting carbon fibers is less than $30 \mathrm{~V}$, and the number of carbon fibers is more than 80 . For instance, if the potential drop in the carbon fibers is assumed to be $20 \mathrm{~V}$ to $10 \mathrm{~V}$, the 
number of carbon fibers is estimated to be 120 to 240 . In this calculation, if the length of the short-circuiting carbon fibers is $600 \mu \mathrm{m}$, twice as long as the $300 \mu \mathrm{m}$ estimated above, the necessary number of carbon fibers to cause the same potential drop is also twofold, as shown in figure 8.

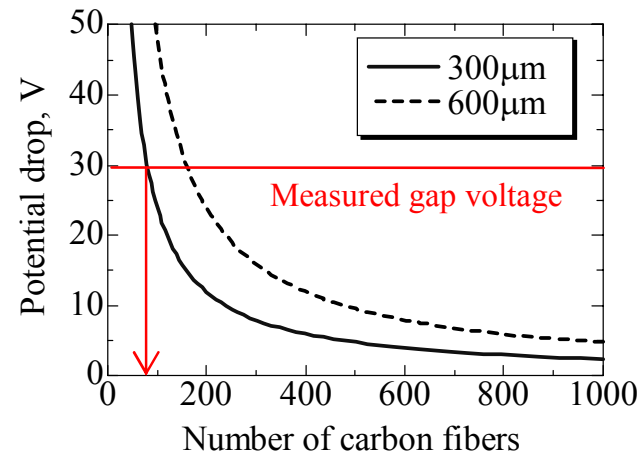

Fig. 8 Calculated result of potential drop in carbon fibers

\subsection{Estimation using Temperature of Short-Circuiting Carbon Fibers}

Next, the relationship between the number of short-circuiting carbon fibers and the temperature of the fibers in short-circuiting is investigated in order to ascertain the necessary number of carbon fibers to cause the phenomena shown in figure 1(a) in the short-circuiting pulse. In this calculation, the density and specific heat of carbon fibers are assumed to be $1,760 \mathrm{~kg} / \mathrm{m}^{3}$ and $754 \mathrm{~J} / \mathrm{kg} \cdot \mathrm{K}$, respectively ${ }^{(8)}$. All of the Joule heat generated in carbon fibers is assumed to be consumed in the temperature rise of the fibers without conduction to the CFRP body. Figure 11 shows the calculated temperature. If carbon fibers are not removed by the short-circuiting, the temperature of the fibers must be less than their $3,600{ }^{\circ} \mathrm{C}$ sublimation point ${ }^{(9)}$. This condition gives the minimum number of carbon fibers as 150 . If heat conduction from the carbon fibers to the CFRP body and the heat capacity of matrix resin are taken into account, the minimum number of carbon fibers would be less than that estimated above. On the other hand, the length of the short-circuiting carbon fiber does not affect the estimated results.

Next, the maximum number of carbon fibers will be obtained under the condition that intense light was observed in figure 1(a). If the intense light shown in figure 1(a) is emitted not from arc discharge plasma but from high-temperature carbon fibers, the temperature of the fibers is thought to be above $1,000{ }^{\circ} \mathrm{C}$. On the basis of this condition, the upper limit of the number of short-circuiting carbon fibers can be estimated to be 260, as shown in figure 9 .

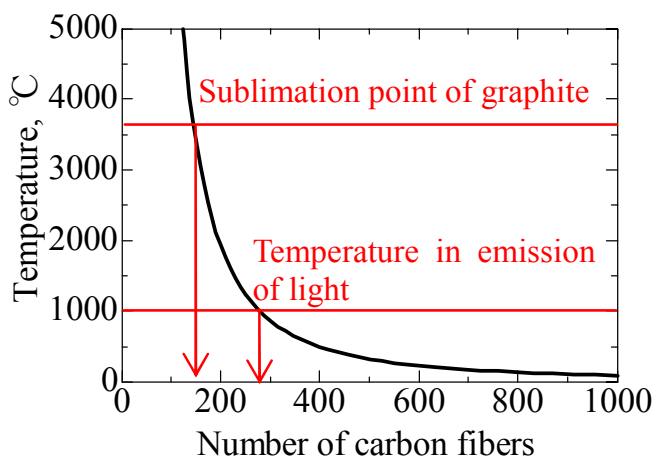

Fig. 9: Calculated result of temperature of carbon fibers 


\subsection{Discussion}

Consequently, it was found that the estimated gap voltage is consistent with the measured one when the phenomenon shown in figure 1(a) is considered to be short-circuiting due to the frayed carbon fibers. It was also found that the number of the carbon fibers in short-circuiting determined from the condition concerning the gap voltage is 80 to 260 , which coincides with the number derived from the condition concerning temperature. Therefore, the temperature of the fibers can be estimated to be 1000 to $3600{ }^{\circ} \mathrm{C}$, which is much higher than the melting point of the matrix resin. These estimations indicate that the short-circuiting contributes to material removal in EDM of CFRP.

\section{Conclusion}

We investigated the effect of short-circuiting in order to discuss material removal in EDM of CFRP. The main results obtained are as follows.

1). Short-circuiting due to frayed carbon fibers frequently occurs in sinking EDM of CFRP. The ratio of short-circuiting pulses to total pulses is varied from $10 \%$ to $60 \%$ under the machining conditions used in this work.

2). Short-circuiting caused due to frayed carbon fibers contributes to material removal in EDM of CFRP.

\section{References}

(1) Tsao C.-C., 2008, Thrust force and delamination of core-saw drill during drilling of carbon fiber reinforced plastics (CFRP), Int. J. Adv Manuf Technol, 37, pp 23-28.

(2) Larsson, E., Eriksson, D., Rydberg, P., 2010, Tool wear compensation, SAE International Journal of Aerospace, 2, pp 199-204.

(3) Fukuzawa Y., Mohri N. Tani T., 1997, Electrical discharge machining phenomena of insulating sialon ceramics with an assisting electrode. IJEM, 2, pp 25-30.

(4) Itoh A., Hayakawa S., Itoigawa F., Nakamura T., 2010, Feasibility Study on Electrical Discharge Machining of Carbon Fiber Reinforced Plastics and Observation of Machining Phenomena. JJSPE, 77, pp 1140-1145 (in Japanese).

(5) Wang H., Habib S., Okada A., Uno U., 2010, EDM Characteristics of Carbon Fiber Reinforced Plastic, Proc. 16th ISEM, pp 65-68.

(6) Saeki T., Kunieda M., Ueki M., Satoh Y., 1996, Transient Workpiece Temperature Analysis in the EDM Processes of High Electric Resistance Materials Considering Joule Heating, JJSPE, 62, pp 443-447 (in Japanese).

(7) Kunieda M., Yanatori K., 1997, Study on Debris Movement in EDM Gap, IJEM, 2, pp 43-49.

(8) Toray industries inc., TORAYCA milled fiber technical reference.

(9) Ermolenko I. N., Lyubliner I. P., Gulko N. V., 1990, Chemically Modified Carbon Fibers and Their Applications, VCH, p 31. 